\title{
Anabaena Sensory Rhodopsin: Effect of point mutations on PSBR photo-isomerization speed
}

\author{
Damianos Agathangelouํㅜ, Yoelvis Orozco-Gonzalez ${ }^{1}$, Marí del Carmen Marin Pérez², \\ Johanna Brazard ${ }^{1}$, Hideki Kandori ${ }^{3}$, Kwan-Hwang Jung ${ }^{4}$, Jérémie Léonard ${ }^{1}$, Nicolas \\ Ferré5, Massimo Olivucci ${ }^{2,6}$, and S.Haacke ${ }^{1, *}$ \\ ${ }^{I}$ Université de Strasbourg - CNRS UMR 7504, Institut de Physique et Chimie des Matériaux de \\ Strasbourg, Strasbourg 67034, France \\ ${ }^{2}$ Department of Biotechnology, Chemistry \& Pharmacy, Università di Siena, 2, I-53100 Siena, Italy \\ ${ }^{3}$ Department of Frontiers Materials, Nagoya Institute of Technology, Showa-ku, Nagoya 466-8555, \\ Japan \\ ${ }_{5}^{4}$ Department of Life Science and Institute of Biological Interfaces, Sogang University, South Korea \\ ${ }^{5}$ Aix-Marseille Université, CNRS, ICR, 13284 Marseille, France \\ ${ }^{6}$ Department of Chemistry, Bowling Green State University, Bowling Green, Ohio 43403, United \\ States
}

\begin{abstract}
We report new experimental results on the ultrafast photoisomerization of ASR - PSBR where, unlike other retinal proteins, point mutations lead to a 2-fold increase of the photo-isomerization speed for the all-trans isomer. Prominent low-frequency vibrational coherences are reported for both the excited and photo-product ground states.
\end{abstract}

\section{Introduction}

Anabaena sensory rhodopsin (ASR) is a microbial retinal protein for which the protonated Schiff base of retinal (PSBR) adopts two different conformations in the ground state, the all-trans,15-anti (AT) and 13-cis, 15-syn (13C). Related to the biological function of ASR being a light-intensity sensor, the isomer ratio depends upon illumination conditions (intensity and wavelength) reaching a photostationary, socalled light adapted (LA) state. Interestingly, upon incubation in the dark at room temperature, the AT population exceeds $97 \%$, defining the so-called dark-adapted (DA) state. AT and 13C undergo two distinct photo-cycles interconverting on a millisecond time scale [1].

The interest of studying the primary steps of retinal proteins' photo-cycle via femtosecond spectroscopy originates from a long-standing question, on how the protein environment tunes and optimizes the photoisomerization reaction speed and yield. In this context, ASR is a particularly suitable model system, since it allows to study the photoreaction of both conformers in the same protein environment at the same time. In particular, the excited state lifetime (ESL) is representative of the

\footnotetext{
*Corresponding author : haacke@unistra.fr
} 
photoisomerization speed. In the wild-type protein (WT-ASR), it is only $150 \mathrm{fs}$ for $13 \mathrm{C}$, and $\sim 5$ times longer for AT (750fs). The relative isomerization quantum yield (QY) is 2.7 times higher for the AT isomer [2,3]. However, the absolute QY values are the lowest ever reported for retinal proteins $(<25 \%)$ demanding an extended study on point mutated proteins for a deeper understanding.
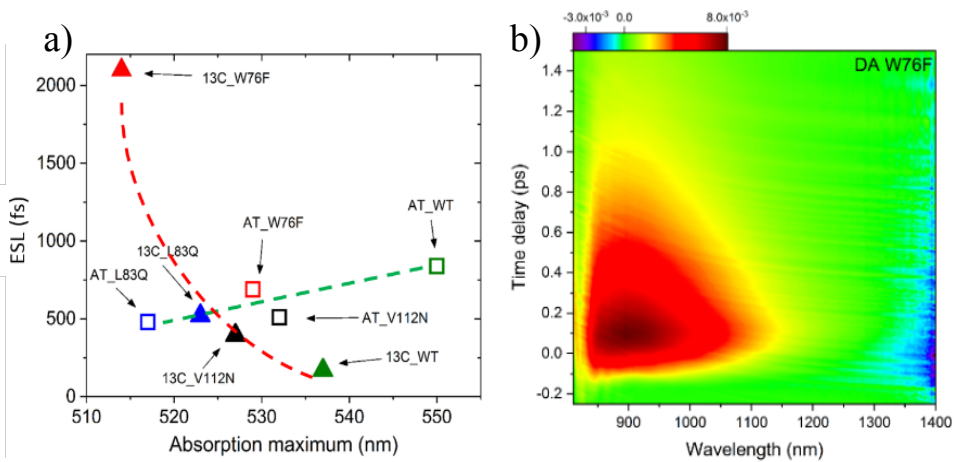

Fig. 1: a) Excited state lifetimes, as determined from stimulated emission decay with respect to absorption maxima of AT (open squares) and 13C isomers (triangles) in WT-ASR and three mutants; Dashed lines are guides for the eyes. b) Contour map from NIR probing region for AT W76F

We report on point-mutated proteins showing an up to $\sim 2$ fold reduction of the AT isomer ESL with respect to WT-ASR, with a trend indicating a correlation between ESL and absorption maximum wavelength (fig 1a) [4]. In contrary, the 13C isomer ESL appears less sensible to mutations leading in some cases to even longer ESL than in WT-ASR. Prominent oscillatory features in the range $<400 \mathrm{~cm}^{-1}$ are observed along the whole detection spectral range, attributed to vibrational modes driving the photo-isomerization reaction.

\section{Experimental results and discussion}

Transient absorption (TA) experiments were performed with a 1-kHz Ti:Sa amplifier, pumping a home-built NOPA that provides 40fs Fourier-limited excitation pulses in the range of $520-560 \mathrm{~nm}$. A chirped white-light continuum $(320-850 \mathrm{~nm}$ or $800-1400 \mathrm{~nm})$ is produced in a $2 \mathrm{~mm}$ thick $\mathrm{CaF}_{2}$ crystal or in a $6 \mathrm{~mm}$ YAG crystal respectively and used as probe. ASR proteins were solubilized in pH 7.0 buffer $(150 \mathrm{mM} \mathrm{NaCl}, 50 \mathrm{mM}$ Tris-HCL, $0.02 \%$ DDM) and light-adapted by constant illumination of the sample reservoir with a 460nm or $590 \mathrm{~nm}$ LED. Pure AT and 13C spectroscopic signatures were inferred from linear combinations of the DA and LA datasets, knowing the isomer content of each sample from HPLC analysis.

TA experiments probing the UV/VIS part of the spectrum highlighting the prominent excited-state or photoproduct absorptions (ESA and PA) were reported for these mutants [4]. We concentrate here on the near-IR stimulated emission (SE), which allows to retrieve the ESL's without any interfering ground state signal (fig $1 \mathrm{~b}$ ). In all the cases, AT isomers of the mutants absorb at shorter wavelengths in respect with WTASR and show a bi-exponential excited state decay with a dominant $>70 \%$ amplitude on the short time constant. From the fits, we find $(0.50 \pm 0.04) \mathrm{ps}$ and $(1.9 \pm 0.2) \mathrm{ps}$ for AT W76F, $(0.31 \pm 0.04)$ ps and $(1.1 \pm 0.2)$ ps for AT V112N and $(0.28 \pm 0.04)$ ps and 
(1.26 \pm 0.2$)$ ps for AT L83Q. The ESLs are identified by the average decay times, i.e. $(0.69 \pm 0.05)$ ps for $W 76 F,(0.51 \pm 0.05)$ ps for $V 112 \mathrm{~N}$ and $(0.48 \pm 0.05)$ ps for L83Q. The acceleration of the photo-isomerization reaction in blue-shifted mutants suggests a steeper slope of the excited state potential energy surface (PES) than in WT-ASR. This can be tentatively explained by a lifted degeneracy between the $S_{1}$ and $S_{2}$ states upon mutations, in contrast to AT WT-ASR where electronic mixing between $S_{1}$ and $S_{2}$ is more prominent, thus inducing a flat PES in the vicinity of the Franck-Condon region [5]. A detailed QM/MM simulation is in progress.
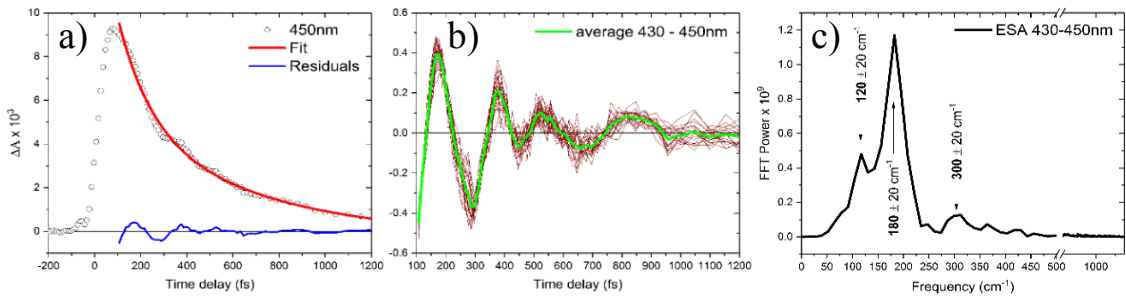

Fig. 2. a) ESA kinetic trace of AT-W76F at $450 \mathrm{~nm}$ b) Residuals after fit over a 20nm range of individual ESA kinetic traces in AT W76F; green line presents the average c) Power spectrum of the residuals of fig (b).

Oscillatory signatures of active vibrational modes in the excited and ground state show up in the TA data for all mutants (fig. 2), but their functional role in the excited state reaction is unclear. Fig. 2a shows a kinetic trace at a central ESA wavelength of AT W76F where the oscillations are $>4$ times above the noise floor. These are isolated by subtracting a fitting double exponential decay from the data (fig $2 \mathrm{a}$ ). Exploring a $20 \mathrm{~nm}$ (430-450nm) broad range (fig 2b), the residuals from individual wavelength fits overlay (brown lines). An FFT of the averaged residuals (green line) results in the power spectrum shown in Fig 2c with dominant contributions at frequencies of $120 \mathrm{~cm}^{-1}, 180 \mathrm{~cm}^{-1}$ and $300 \mathrm{~cm}^{-1}$ in agreement with previous reports for the stimulated emission of ASR and BR [6]. For AT W76F, the central $180 \pm 20 \mathrm{~cm}^{-1}$ mode is the dominant excited state mode. Additionally, a similar data treatment for the PA band (not shown) reveals a dominant $80 \mathrm{~cm}^{-1}$ mode attributed to ground oscillations induced by the ultrafast $S_{1}$ decay, analogous to those reported for rhodopsin-mimicking molecular photo-switches [7].

[1] A. Kawanabe and H. Kandori, Sensors, 9, 9741-9804(2009)

[2] A. Wand, R. Rozin, T. Eliash, K-H. Jung, M. Sheves, S. Ruhman, ACS, 133, 20922-20932 (2011)

[3] A. Cheminal, J. Léonard, S. Kim, K-H. Jung, H. Kandori, S. Haacke, PCCP, 17, 2542925439 (2015)

[4] D. Agathangelou, Y. Orozco-Gonzalez, M. del Carmen Marin, P.P. Roy, J. Brazard, H. Kandori, K.-H Jung, J. Léonard, T. Buckup, N. Ferré, M. Olivucci, S. Haacke, Faraday. Disc. 207, 55-75 (2018).

[5] M. Manathunga, X. Yang, Y. Orozco-Gonzalez, M. Olivucci, J. Phys. Chem. Lett., 8, 5222-5227 (2017).

[6] A. Wand, B. Loevsky, N. Friedman, M. Sheves, S. Ruhman, J. Phys. Chem. B, 117, 46704679 (2013).

[7] M. Gueye, M. Manathunga, D. Agathangelou, Y. Orozco, M. Paolino, S. Fusi, S. Haacke, M. Olivucci, J. Léonard, Nat. Comm, 9:313, 1-8 (2018). 